\title{
Significance of BRAF V600E Mutation and Cytomorphological Features for the Optimization of Papillary Thyroid Cancer Diagnostics in Cytologically Indeterminate Thyroid Nodules
}

\author{
Authors \\ Augustas Beiša1, 2, Mindaugas Kvietkauskas1, 2, Virgilijus Beiša1, 2, Mindaugas Stoškus¹, 2, Elvyra Ostanevičiūtė1, 2, \\ Eugenijus Jasiūnas ${ }^{3}$, Laimonas Griškevičius ${ }^{1,2}$, Dmitrij Šeinin ${ }^{4}$, Auksẻ Šileikytè1, Kęstutis Strupas ${ }^{1,2}$
}

\author{
Affiliations \\ 1 Vilnius University Faculty of Medicine \\ 2 Vilnius University Hospital Santaros Klinikos \\ 3 Mykolas Romeris University \\ 4 National Center of Pathology, Affiliate of Vilnius \\ University Hospital Santaros Klinikos
}

Key words

Bethesda system, BRAF mutation, fine-needle aspiration, papillary thyroid cancer, thyroid

$\begin{array}{ll}\text { received } & 25.11 .2017 \\ \text { revised } & 18.02 .2018 \\ \text { accepted } & 08.03 .2018\end{array}$

Bibliography

DOI https://doi.org/10.1055/a-0588-4885

Published online: 22.3.2018

Exp Clin Endocrinol Diabetes 2019; 127: 247-254

(c) J. A. Barth Verlag in Georg Thieme Verlag KG Stuttgart .

New York

ISSN 0947-7349

Correspondence

Augustas Beiša

Vilnius University Faculty of Medicine

M. K. Čiurlionio str. 21

LT-03101 Vilnius, Lithuania

Tel.: + 370/85/239 8700, Fax: + 370/85/2398705

augustas.beisa@santa.It

\section{ABSTRACT}

Background Ultrasound guided fine needle aspiration biopsy with cytologic analysis is an initial step in diagnostic of thyroid nodules. Unfortunately, up to $30 \%$ of biopsies are indeterminate and diagnostic surgery is required. The aim of this study was to estimate the diagnostic value of BRAF V600E mutation status combined with cytomorphological features for diagnosis of papillary thyroid cancer (PTC) in cytologically indeterminate thyroid nodules.

Methods A prospective study analyzed patients who had ultrasound suspicious thyroid nodules, underwent fine needle aspiration and cytological examination, and were classified according to the Bethesda system. Patients from indeterminate diagnostic categories were examined for BRAF V600E mutation and 22 cytomorphological features, and underwent thyroid surgery. A binary logistic regression model was used to evaluate the diagnostic utility.

Results A total of 219 patients met study criteria. After histological examination, 77 (35.2\%) patients were diagnosed with PTC and 142 (64.8\%) with benign nodular thyroid disease. According to logistic regression model, significant features for PTC diagnosis were: liquid colloid consistency, papillary structures, eosinophilic colloid bodies, and BRAF V600E mutation. Risk groups classified by this model have sensitivity of $80.5 \%$ ( $95 \%$ Cl: 69.9 to 88.7), specificity of $99.3 \%$ (95\% Cl: 96.1 to $100)$, positive predictive value of $98.4 \%$ ( $95 \% \mathrm{Cl}: 89.8$ to 99.8$)$, negative predictive value of $90.4 \%(95 \% \mathrm{Cl}: 85.7$ to 93.7$)$, and accuracy of $92.7 \%$ (95\% Cl: 88.4 to 95.8 ) for PTC diagnosis. Conclusions Evaluation of BRAF V600E mutation status combined with cytomorphological features for diagnosis of PTC in cytologically indeterminate thyroid nodules can significantly improve diagnostic accuracy and reduce the number of diagnostic operations (calculator available at www.ptc-calc. we2host.lt).

\section{Introduction}

Nodular thyroid disease is one of the most frequently diagnosed pathologies. Some studies have shown that up to $70 \%$ of randomly selected individuals have nodular disease, which is more common in women and among older people [1-3]. A key challenge for clinicians is to decide which nodules require evaluation and inter- vention [4]. Although thyroid cancer (TC) only accounts for approximately $7-15 \%$ of all ultrasound (US) detected nodules, the incidence of TC, particularly papillary thyroid carcinoma (PTC), has increased dramatically in many countries over the past few decades $[3,5]$. This can be explained by increased use of a high sensitivity diagnostic methods and medical radiation, iodine intake, carcino- 
gens, elevated background radiation, environmental, ethnic and genetic factors or combinations of these factors [6].

The fine needle aspiration (FNA) biopsy performed under US guidance remains the gold standard diagnostic test for the initial evaluation of a thyroid nodule. Reported sensitivity and specificity for US-guided FNA range from 65 to $98 \%$ and 72 to $100 \%$, respectively [7]. FNA biopsy specimens are classified according to the Bethesda System for Reporting Thyroid Cytopathology into the 6 categories. Each of the categories has an implied cancer risk that links it to a rational clinical management guideline [8]. Due to the well-differentiated nature of most TCs and their morphologic overlap with benign nodules, indeterminate diagnostic categories, Atypia of Undetermined Significance/ Follicular Lesion of Undetermined Significance (AUS/FLUS), Follicular Neoplasm/ Suspicious for a Follicular Neoplasm (FN/SFN), and Suspicious for Malignancy (SUSP), comprise 10-26\% of all cytology examinations [9]. According to Ali and Cibas estimated risk of malignancy varies from 5 to $15 \%$ for AUS/FLUS, 15 to $30 \%$ for FN/SFN, and 60 to $75 \%$ for SUSP category [8]. Due to malignancy risk cannot be excluded in indeterminate cases, surgery is typically recommended; however, up to two-thirds of these cases are found to be benign at the time of surgical resection [10]. This leads to unnecessary surgical procedures, complications, and morbidity, as well as increased health care expenses.

As the preoperative PTC diagnostic criteria in indeterminate diagnostic categories remain unclear, the main goal of recent studies is to improve the accuracy of FNA biopsy assessment and to predict tumor behavior.

The aim of this study was to estimate the diagnostic value of BRAF V600E mutation status combined with cytomorphological features for diagnosis of PTC in cytologically indeterminate thyroid nodules.

\section{Material and methods}

A prospective study was performed between January 2012 and February 2016. All patients with a US-suspicious thyroid nodule underwent an US-guided FNA biopsy in Vilnius University Hospital Santaros Klinikos. Twenty-two cytomorphological features were analyzed. Patients were classified using Bethesda System for Reporting Thyroid Cytopathology [8]. Patients diagnosed with AUS/FLUS, FN/ SFN, or SUSP were considered as the objects of this study and were involved in further investigation. Cytology specimens of these patients were analyzed for the BRAF V600E mutation. Patients who refused operation or were operated in other Clinics were excluded from further investigation. Operated patients diagnosed with TC on intraoperative biopsy, underwent total thyroidectomy with prophylactic unilateral or bilateral central lymph node dissection (of level $\mathrm{VI}$ ) according to tumor localization. In addition, patients who preoperatively had suspicious lymph nodes on US, underwent modified radical lymph node dissection (of level II-V). The decision on the extension of the operation was not influenced by genetic investigation results. Surgically removed thyroid tissue was sent for routine histological examination and additional analysis of the BRAF V600E mutation for patients diagnosed with PTC in whom mutation was not detected in the initial FNA biopsy cytology specimen. All patients diagnosed with a PTC or benign nodular thyroid diseas- es were selected for further investigation. Detailed inclusion and exclusion criteria are presented in $>$ Fig. 1.

\section{Fine needle aspiration biopsy cytological analysis}

Every aspirate was transferred to a liquid-based preparation for routine cytological assessment. Smears stained with Papanicolaou stain and automatic BD PrepStain system were evaluated by one experienced cytologist. Bright-field microscope was used. Initially, whole preparation was examined with 10x magnifying lens. Presence of at least 6 groups of thyroid epithelial cells, with each group containing 7-10 cells satisfies the adequacy of the aspirate. Cytomorphological features including morphology of nuclei, anisokaryosis expression, chromatin morphology (homogenity, striation, and dispersion), presence of nucleoli, amount and intensity of cytoplasm, presence of degeneration features, Hürthle cells, lymphocyte, macrophage infiltration, normofollicular/macrofollicular, microfollicular structures, papillary structures, atypia, intranuclear cytoplasmic inclusions, psammoma bodies, amount and consistency of colloid, and presence of eosinophilic colloid bodies were analyzed with 40x or $100 x$ magnifying lenses. Patients were classified using the Bethesda System for Reporting Thyroid Cytopathology [8].

\section{BRAF V600E mutation analysis}

The method of genetic investigation of the BRAF V600E mutation was previously described by Beisa et al. [11]. Material from the liquid-based preparation (treated with RBC lysis solution) or histology specimen was transferred into 1.5-ml tubes. Genomic DNA was extracted with the GeneJET Genomic DNA Purification Kit (Thermo Scientific, Vilnius, Lithuania). In order to detect the BRAF V600E mutation (GTG > GAG), we developed a Real-Time PCR assay comprised of two types of reactions. The first one served as an internal reference sample control flanking the BRAF codon V600 of the BRAF gene. The second reaction, or the mutation specific reaction, was designed to specifically target the GTG > GAG mutation. Primers were predesigned manually and investigated in silico using the OligoCalc web tool. A BRAF V600E mutation specific forward primer was edited manually by introducing a relevant mismatch nucleotide at the 3' end according to the Amplification Refractory Mutation System primer design approach. Both reactions shared the same reverse primer. Primer sequences were as follows: BRAF_ V600E_Forward gtgattttggtctagctacggag; BRAF_Forward taggtgattttggtctagctacag; BRAF_Reverse CATCCACAAAATGGATCCAGAC. Real-Time PCR reactions were performed in duplicates using a Maxima ${ }^{\mathrm{TM}}$ SYBR Green qPCR Master Mix supplemented with 0.4 units per reaction of UNG (both Thermo Scientific, Vilnius, Lithuania), $300 \mathrm{nmol}$ of each primer (Metabion, 82152 Planegg/ Steinkirchen, Germany), and $0.005-0.05 \mu \mathrm{g}$ of DNA in a $20 \mu \mathrm{l}$ reaction volume. Real-Time PCR reaction conditions on a Bio-Rad CFX96 system (Bio-Rad Laboratories, Hercules, CA, USA) were as follows: 2 min at $50^{\circ} \mathrm{C}, 10 \mathrm{~min}$ at $95^{\circ} \mathrm{C}, 37$ cycles at $95^{\circ} \mathrm{C}$ for $15 \mathrm{~s}$, and $1 \mathrm{~min}$ at $60^{\circ} \mathrm{C}$. The first $20 \mathrm{BRAF}$ V600E-positive and 20 -negative cases were confirmed by sequencing the histology specimens and a sequencing assay published elsewhere. The sensitivity of the RealTime PCR assay was tested by serial dilutions of previously sequenced highly positive BRAF V600E DNA in negative DNA. The Real-Time PCR assay proved to detect mutant DNA down to a 2.5 log dilution in a wild-type background. 


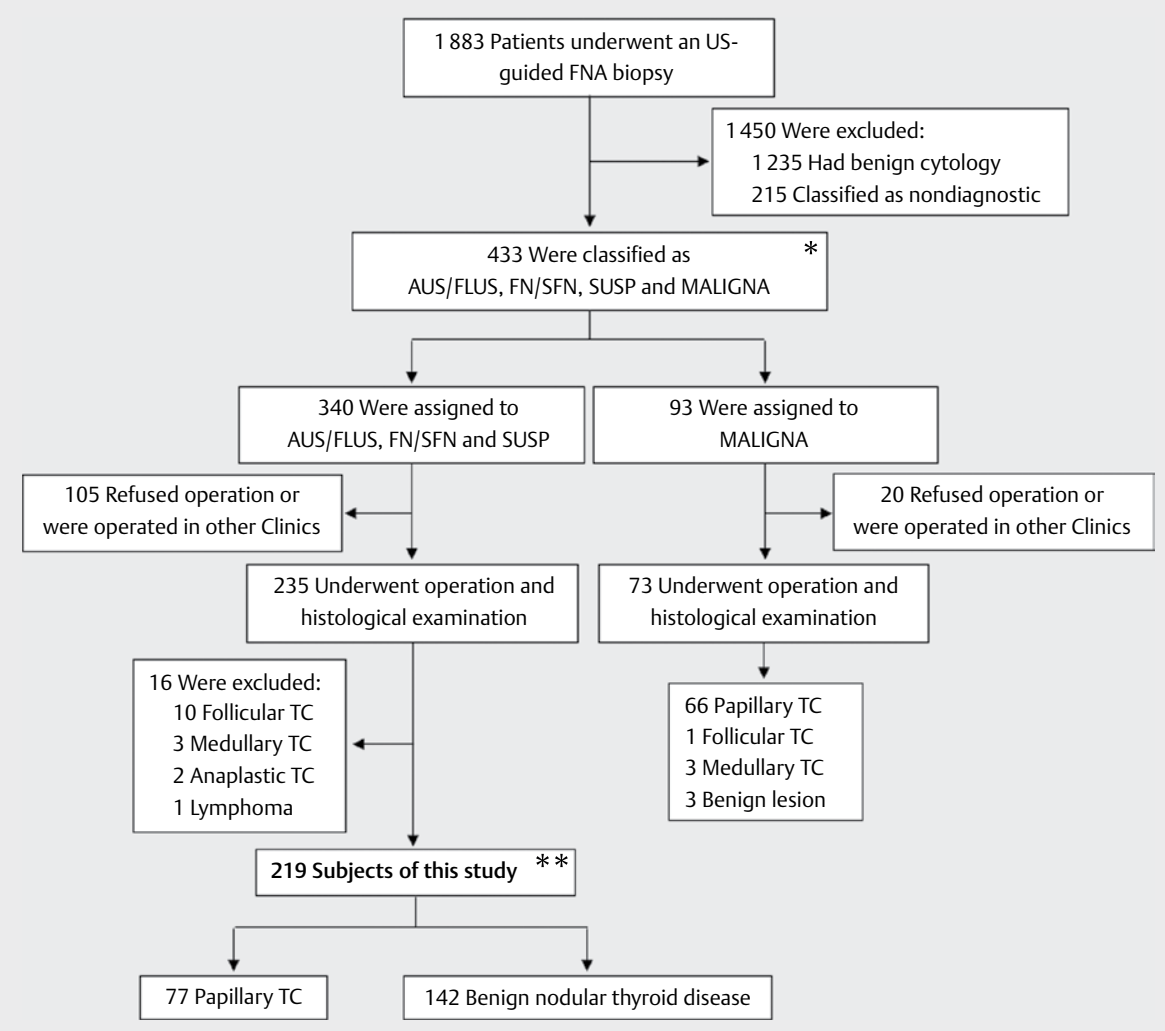

- Fig. 1 Flow diagram of study inclusion and exclusion criteria. * Cytology specimens of these patients were analyzed for the BRAF V600E mutation. ${ }^{*}$ * Histological specimens of patients diagnosed with PTC in whom mutation was not detected in the initial FNA biopsy cytology specimen were additionally analyzed for the BRAF V600E mutation. All non PTC cases were BRAF V600E negative.

\section{Statistical analysis}

All statistical analyses were performed with IBM SPSS STATISTICS, version 23.0. Continuous variables were checked for normal distribution by the Shapiro-Wilk test. Non-parametric data were expressed as medians and ranges, and analyzed by the Mann-Whitney $U$ test. Categorical variables were reported as absolute values and percentages, and analyzed using Pearson Chi-Square test or Fisher's Exact Test. A p-value less than 0.05 at $95 \%$ confidence interval was considered statistically significant.

To evaluate the diagnostic value of the preoperative data, including gender, age, cytomorphological features, Bethesda categories, and status of BRAF V600E mutation, for PTC diagnosis, a binary logistic regression model was used.

Mathematically, logistic regression was expressed by the equation:

$$
\mathrm{Z}=\ln \frac{\mathrm{P}(\mathrm{Y}=1)}{\mathrm{P}(\mathrm{Y}=0)}=\mathrm{C}+\mathrm{b}_{1} \mathrm{x}_{1}+\mathrm{b}_{2} \mathrm{x}_{2}+\mathrm{b}_{3} \mathrm{x}_{3}+\ldots+\mathrm{b}_{n} \mathrm{x}_{n}
$$

Consequently, the probability of the event occurring $P(Y=1)$ (diagnosis of PTC is true) was defined as follows:

$\mathrm{P}(\mathrm{Y}=1)=\frac{\mathrm{e}^{\mathrm{Z}}}{1+\mathrm{e}^{\mathrm{Z}}}=\frac{1}{1+\mathrm{e}^{-\mathrm{Z}}}$
And the probability of the event not occurring $\mathrm{P}(\mathrm{Y}=0)$ (diagnosis of PTC is false) was defined as follows:

$$
\mathrm{P}(\mathrm{Y}=0)=1-\mathrm{P}(\mathrm{Y}=1)
$$

In this model $\ln \mathrm{P}(\mathrm{Y}=1) / \mathrm{P}(\mathrm{Y}=0)$ is the logit function of probability ratio $P(Y=1) / P(Y=0)$. C is the intercept parameter, and $b_{1}, b_{2}$, $b_{3}, \ldots, b_{n}$ are the slope parameters of regression equation. Accordingly, $c, b_{1}, b_{2}, b_{3}, \ldots, b_{n}$ are the regression equation coefficients, which were obtained using data of study sample and which represent the values of how the criterion variables change when the predictor variables change. $X_{1}, X_{2}, X_{3}, \ldots, X_{n}$ are the independent explanatory criterions predictor variables, which can gain value of 0 (if the feature is absent) or 1 (if the feature is present). The symbol "e" represents natural logarithm base $(\mathrm{e} \approx 2,7182818284 \ldots$...).

For assessment of the logistic regression model pertinence for the data of study sample, were used: the Hosmer-Lemeshow goodness-of-fit test (to assess the model compatibility with data of study sample), the classification table (to evaluate the predictive accuracy of the model), the Nagelkerke $\mathrm{R}^{2}$ ( $\mathrm{R}$ square) pseudo-determinations coefficient (to assess the efficiency of the model), and Wald $\mathrm{X} 2$ statistics (to assess the significance of corresponding coefficients in this model).

The cutoff of 0.5 was selected and patients were divided into two groups of PTC risk: low risk group (when PTC risk<0.5) and high risk group (when PTC risk $\geq 0.5$ ). 
To assess the diagnostic characteristics of a model, cases were sorted by the risk of PTC diagnosis and results of the final histological examination into true positives (when risk of PTC diagnosis was high and after final histological examination PTC was confirmed), true negatives (when risk of PTC diagnosis was low and after final histological examination benign nodular thyroid disease was confirmed), false positives (when risk of PTC diagnosis was high and after final histological examination benign nodular thyroid disease was confirmed), and false negatives (when risk of PTC diagnosis was low and after final histological examination PTC was confirmed).

The diagnostic characteristics of the model were expressed with sensitivity, specificity, positive predictive value, negative predictive value, and accuracy with their $95 \%$ confidence intervals.

\section{Results}

\section{General characteristics of patients}

A total of 1883 patients underwent an US-guided FNA biopsy. Three hundred forty patients were classified as AUS/FLUS, FN/SFN, and SUSP. Two hundred thirty-five of these patients underwent thyroid surgery in our Clinics due to suspected malignancy. Sixteen patients were excluded from this study: 10 diagnosed with follicular TC, 3 with medullary TC, 2 with anaplastic TC, and 1 with lymphoma.

A total of 219 patients were involved in this study for further investigation. Out of them, 188 (85.8\%) were female and 31 (14.2\%) - male. The mean age of the patients was $54.3 \pm 13.4$ years. After a cytological investigation, 42 (19.2\%) out of the 219 FNA biopsies were classified as AUS/FLUS, 126 (57.5\%) as FN/SFN, and 51 (23.3\%) as SUSP. Histological investigation of the surgically removed thyroid tissue revealed 77 PTC cases: 14 (33.3\%) patients from AUS/ FLUS category, 22 (17.5\%) from FN/SFN, and 41 (80.4\%) from SUSP. Out of all PTC patients, 55 (71.4\%) had classical type of PTC, 19 (24.7\%) had follicular type, and 3 (3.9\%) had oncocytic type.

Based on the results of the histological examination, the patients were divided into two comparative groups: 77 (35.2\%) - the PTC group, and 142 (64.8\%) - the group of benign nodular thyroid disease. Out of 77 patients of PTC group, 71 (92.2\%) were female and $6(7.8 \%)$ - male, and the mean age of this group was $53.6 \pm 12.4$ years. Out of 142 patients diagnosed with benign nodular thyroid disease, 117 (82.4\%) were female and 25 (17.6\%) - male, and the mean age of this group was $54.6 \pm 14$ years. Female gender was significantly related to PTC diagnosis $(p=0.047)$. There was no statistically significant difference between these two groups when comparing the mean age $(p=0.405)$.

BRAF V600E mutation in the FNA cytological specimen was found for 37 (48.1\%) patients from PTC group and for 0 patients from benign nodular thyroid disease group. After the additional genetic investigation of the postoperative histological specimens in cytologically BRAF V600E-negative PTC cases, 15 additional BRAF V600E positive cases were identified. A total of 52 (67.5\%) PTC cases were positive for the BRAF V600E mutation and 25 (32.5\%) were negative. All patients from the benign group were BRAF V600E negative. The molecular analysis results of the cytology and histology specimens revealed that in PTC group were statistically significantly more patients with BRAF V600E mutation comparing with benign nodular thyroid disease group $(p=0.000)$.
Cytomorphological features were compared between PTC and benign nodular thyroid disease groups. The presence of high anisokaryosis ( $18.2 \%$ vs. $8.5 \%, p=0.034)$, streaked chromatin $(68.8 \%$ vs. $37.3 \%, p=0.000)$, dark cytoplasm ( $44.2 \%$ vs. $19 \%, p=0.000)$, lymphocyte infiltration ( $63.6 \%$ vs. $49.3 \%, p=0.042)$, papillary structures ( $50.6 \%$ vs. $8.5 \%, p=0.000)$, atypia ( $96.1 \%$ vs. $76.1 \%$, $p=0.000)$, intranuclear cytoplasmic inclusions ( $55.8 \%$ vs. $10.6 \%$, $p=0.000)$, psammoma bodies $(10.4 \%$ vs. $1.4 \%, p=0.004)$, densely colloid ( $45.5 \%$ vs. $12 \%, p=0.000)$, and eosinophilic colloid bodies $(29.9 \%$ vs. $13.4 \%, p=0.003)$ were shown to be significantly related to PTC. Lesions with homogenous chromatin (62.3\% vs. $91.5 \%, p=0.000)$, light cytoplasm ( $55.8 \%$ vs. $81 \%, p=0.000)$, prominent amount of colloid ( $6.5 \%$ vs. $16.2 \%, p=0.040)$, and liquid colloid ( $33.8 \%$ vs. $73.2 \%$ ) were shown to be significantly related to benign nodular thyroid disease. Nuclei morphology, presence of coarsely and finely dispersed chromatin, nucleoli, degeneration, Hürthle cells, macrophage infiltration, mormofollicular/macrofolicular, and amount of cytoplasm demonstrated statistically insignificant differences between groups. Detailed data are presented in $>$ Table 1.

\section{Diagnostic value of BRAF V600E mutation status combined with cytomorphological features for PTC diagnosis}

According to our logistic regression model, we determined that out of all preoperative data (gender, age, cytomorphological features, Bethesda categories) and status of BRAF V600E mutation significant features for PTC diagnosis were: liquid colloid consistency (this feature is defined by the variable $X_{1}$ in the logistic equation and its coefficient is -1.46, which shows an indirect dependency of PTC diagnosis), papillary structures (this feature is defined by the variable $\mathrm{X}_{2}$ in the logistic equation and its coefficient is 2.16, which shows a direct dependency of PTC diagnosis), eosinophilic colloid bodies (this feature is defined by the variable $X_{3}$ in the logistic equation and its coefficient is 1.57, which shows a direct dependency of PTC diagnosis), and BRAF V600E mutation (this feature is defined by the variable $\mathrm{X}_{4}$ in the logistic equation and its coefficient is 22.79 , which shows a direct dependency of PTC diagnosis). Other features, as shown by our logistic regression based model, do not have a significant effect on histological examination results (adding an additional variable does not improve the model). The logistic regression model was expressed by logit function of probability ratio $z$ :

$Z=(-1.82)+(-1.46) \times($ liquid colloid consistency $)+2.16 \times$ (papillary structures) $+1.57 \times$ (eosinophilic colloid bodies $)+$ $22.79 \times$ (BRAF V600E mutation).

The Hosmer-Lemeshow test produced a p-value of 0.998 . Nagelkerke's $R^{2}$ is equal to 0.76 . According to Wald's criterion, all regressors are statistically significant, except for BRAF V600E mutation, which has been exempted due to the high significance for the final results of the logistic regression model.

Out of 219 patients, the regression analysis classified 156 (71.2\%) as having a low risk of PTC, and 63 (28.8\%) patients as high risk of PTC diagnosis. In low risk group, there were 15 (9.6\%) histologically confirmed PTC cases and 141 (90.4\%) - benign nodular thyroid disease cases. In high risk group, there were 62 (98.4\%) and $1(1.6 \%)$ cases, respectively. 
- Table 1 Cytomorphological features statistical comparison in two comparative groups, n=219.

\begin{tabular}{|c|c|c|c|}
\hline & $\begin{array}{l}\text { Papillary thyroid cancer, } \\
\qquad \mathrm{n}=77\end{array}$ & $\begin{array}{c}\text { Benign nodular thyroid } \\
\text { disease, } n=142\end{array}$ & Value of $p$ Asymp. Sig.(2-sided) \\
\hline \multicolumn{4}{|l|}{ Nuclei (n) } \\
\hline Monomorphic & $1(1.3 \%)$ & $1(0.7 \%)$ & $1^{* * *}$ \\
\hline Polymorphic & $76(98.7 \%)$ & $141(99.3 \%)$ & \\
\hline \multicolumn{4}{|l|}{ Anisokaryosis (n) } \\
\hline None & $0(0 \%)$ & $2(1.4 \%)$ & $0.542 * * *$ \\
\hline Low & $10(13 \%)$ & $35(24.6 \%)$ & $0.041^{*}$ \\
\hline Moderate & $53(68.8 \%)$ & $93(65.5 \%)$ & $0.617^{*}$ \\
\hline High & $14(18.2 \%)$ & $12(8.5 \%)$ & $0.034^{*}$ \\
\hline \multicolumn{4}{|c|}{ Homogenous chromatin (n) } \\
\hline Absent & $29(37.7 \%)$ & $12(8.5 \%)$ & $0.000^{*}$ \\
\hline Present & $48(62.3 \%)$ & $130(91.5 \%)$ & \\
\hline \multicolumn{4}{|c|}{ Streaked chromatin (n) } \\
\hline Absent & $24(31.2 \%)$ & $89(62.7 \%)$ & $0.000^{*}$ \\
\hline Present & $53(68.8 \%)$ & $53(37.3 \%)$ & \\
\hline \multicolumn{4}{|c|}{ Coarsely dispersed chromatin (n) } \\
\hline Absent & $51(66.2 \%)$ & $100(70.4 \%)$ & $0.522^{*}$ \\
\hline Present & $26(33.8 \%)$ & $42(29.6 \%)$ & \\
\hline \multicolumn{4}{|c|}{ Finely dispersed chromatin ( $\mathrm{n}$ ) } \\
\hline Absent & $37(48.1 \%)$ & $86(60.6 \%)$ & $0.075^{*}$ \\
\hline Present & $40(51.9 \%)$ & $56(39.4 \%)$ & \\
\hline \multicolumn{4}{|l|}{ Nucleoli (n) } \\
\hline Absent & $8(10.4 \%)$ & $28(19.7 \%)$ & $0.075^{*}$ \\
\hline Present & $69(89.6 \%)$ & $114(80.3 \%)$ & \\
\hline \multicolumn{4}{|c|}{ Amount of cytoplasm (n) } \\
\hline Sufficient & $47(61 \%)$ & $83(58.5 \%)$ & $0.710^{*}$ \\
\hline Abundant & $30(39 \%)$ & $59(41.5 \%)$ & \\
\hline \multicolumn{4}{|c|}{ Cytoplasm intensity (n) } \\
\hline Light & $43(55.8 \%)$ & $115(81 \%)$ & $0.000^{*}$ \\
\hline Dark & $34(44.2 \%)$ & $27(19 \%)$ & \\
\hline \multicolumn{4}{|l|}{ Degeneration $(n)$} \\
\hline Absent & $37(48.1 \%)$ & $80(56.3 \%)$ & $0.241^{*}$ \\
\hline Present & $40(51.9 \%)$ & $62(43.7 \%)$ & \\
\hline \multicolumn{4}{|l|}{ Hürthle cells (n) } \\
\hline Absent & $57(74 \%)$ & $91(64.1 \%)$ & $0.133^{*}$ \\
\hline Present & $20(26 \%)$ & $51(35.9 \%)$ & \\
\hline \multicolumn{4}{|c|}{ Lymphocyte infiltration (n) } \\
\hline Absent & $28(36.4 \%)$ & $72(50.7 \%)$ & $0.042 *$ \\
\hline Present & $49(63.6 \%)$ & $70(49.3 \%)$ & \\
\hline \multicolumn{4}{|c|}{ Macrophage infiltration (n) } \\
\hline Absent & $28(36.4 \%)$ & $65(45.8 \%)$ & $0.179^{*}$ \\
\hline Present & $49(63.6 \%)$ & $77(54.2 \%)$ & \\
\hline \multicolumn{4}{|c|}{ Normofollicular/Macrofollicular structures (n) } \\
\hline Absent & $51(66.2 \%)$ & $79(55.6 \%)$ & $0.127^{*}$ \\
\hline Present & $26(33.8 \%)$ & $63(44.4 \%)$ & \\
\hline \multicolumn{4}{|c|}{ Microfollicular structures (n) } \\
\hline Absent & $28(36.4 \%)$ & $36(25.4 \%)$ & $0.087^{*}$ \\
\hline Present & $49(63.6 \%)$ & $106(74.6 \%)$ & \\
\hline \multicolumn{4}{|c|}{ Papillary structures (n) } \\
\hline Absent & $38(49.4 \%)$ & $130(91.5 \%)$ & $0.000^{*}$ \\
\hline Present & $39(50.6 \%)$ & $12(8.5 \%)$ & \\
\hline
\end{tabular}


- Table 1 Continued.

\begin{tabular}{|c|c|c|c|}
\hline & $\begin{array}{l}\text { Papillary thyroid cancer, } \\
\qquad \mathrm{n}=77\end{array}$ & $\begin{array}{l}\text { Benign nodular thyroid } \\
\text { disease, } n=142\end{array}$ & Value of $p$ Asymp. Sig.(2-sided) \\
\hline \multicolumn{4}{|l|}{ Atypia (n) } \\
\hline Absent & $3(3.9 \%)$ & $34(23.9 \%)$ & $0.000 * * *$ \\
\hline Present & $74(96.1 \%)$ & $108(76.1 \%)$ & \\
\hline \multicolumn{4}{|c|}{ Intranuclear cytoplasmic inclusions ( $\mathrm{n}$ ) } \\
\hline Absent & $34(44.2 \%)$ & $127(89.4 \%)$ & $0.000^{*}$ \\
\hline Present & $43(55.8 \%)$ & $15(10.6 \%)$ & \\
\hline \multicolumn{4}{|c|}{ Psammoma bodies ( $\mathrm{n}$ ) } \\
\hline Absent & $69(89.6 \%)$ & $140(98.6 \%)$ & $0.004^{* * *}$ \\
\hline Present & $8(10.4 \%)$ & $2(1.4 \%)$ & \\
\hline \multicolumn{4}{|c|}{ Amount of colloid $(n)$} \\
\hline Absent & $16(20.8 \%)$ & $21(14.8 \%)$ & $0.259^{*}$ \\
\hline Occasional & $51(66.2 \%)$ & $79(55.6 \%)$ & $0.127^{*}$ \\
\hline Frequent & $5(6.5 \%)$ & $19(13.4 \%)$ & $0.119^{*}$ \\
\hline Prominent & $5(6.5 \%)$ & $23(16.2 \%)$ & $0.040^{*}$ \\
\hline \multicolumn{4}{|c|}{ Colloid consistency ( $\mathrm{n}$ ) } \\
\hline No colloid & $16(20.8 \%)$ & $21(14.8 \%)$ & $0.259^{*}$ \\
\hline Liquid & $26(33.8 \%)$ & $104(73.2 \%)$ & $0.000^{*}$ \\
\hline Densely & $35(45.5 \%)$ & $17(12 \%)$ & $0.000 *$ \\
\hline \multicolumn{4}{|c|}{ Eosinophilic colloid bodies ( $\mathrm{n}$ ) } \\
\hline Absent & $54(70.1 \%)$ & $123(86.6 \%)$ & $0.003^{*}$ \\
\hline Present & $23(29.9 \%)$ & $19(13.4 \%)$ & \\
\hline
\end{tabular}

According the PTC risk groups classified by our logistic regression model and results of the final histological examination 62 cases were considered as true positive, 141 as true negative, 1 as false positive, and 15 as false negative. PTC risk groups classified by our logistic regression model have sensitivity of $80.5 \%$ (95\% Cl: 69.9 to 88.7 ), specificity of $99.3 \%$ (95\% Cl: 96.1 to 100$)$, positive predictive value of $98.4 \%$ ( $95 \% \mathrm{Cl}: 89.8$ to 99.8 ), negative predictive value of $90.4 \%$ (95\% Cl: 85.7 to 93.7 ), and accuracy of $92.7 \%$ (95\% Cl: 88.4 to 95.8$)$ for PTC diagnosis.

Convenient for clinical use online PTC diagnosis probability calculator for cytologically indeterminate thyroid nodules is available at www.ptc-calc.we2host.lt.

\section{Discussion and conclusions}

Thyroid US with FNA remains the gold standard for evaluation of thyroid nodules [4]. TC accounts only for approximately $7-15 \%$ of all thyroid nodules detected by US [3]. PTC comprises the majority (85-90\%) of all thyroid malignancies [12]. In our study, PTC consisted $87.7 \%$ of all TC cases. In general, PTC has a good prognosis with 25 -year rates of cause-specific mortality about $5 \%$ after appropriate treatment [13]. However, 20 to $30 \%$ of patients develop local recurrence or distant metastasis and $1 \%$ die from PTC [14]. Due to increasing prevalence of thyroid nodules and the lack of specific diagnostic tests for TC, accurate clinical diagnosis prior to surgery became an important challenge to physicians $[1,15]$.
Thyroid US is a widespread technique that is used as a first-line diagnostic procedure for detecting and characterizing nodular thyroid disease [16]. It is indisputable that US-guided FNA is a safe, cost-effective, and reliable diagnostic method in the differential diagnosis of variable thyroid nodules. The Bethesda System for Reporting Thyroid Cytopathology classifies FNA biopsy specimens into 6 categories, each with certain degree of probability for malignancy, guiding the standard-of-care for patients with thyroid nodules [17]. However, some cytologic results such as indeterminate create confusion for clinicians in thyroid nodule management [18]. Indeterminate diagnostic categories comprise $10-26 \%$ of all cytology examinations [9]. About $50 \%$ patients undergo thyroid surgery due to cytologically indeterminate nodules; however, onethird of these cases are found to be malignant at the time of histological examination [19]. In our study, about $76.3 \%$ patients underwent surgery due to indeterminate cytologically and $39.6 \%$ of them were finally diagnosed with TC. According to Ali and Cibas estimated risk of malignancy varies from 5 to $15 \%$ for AUS/FLUS, 15 to $30 \%$ for FN/SFN, and 60 to $75 \%$ for SUSP category [8]. According to our data, the malignancy rate of the AUS/FLUS category was $36.4 \%$, FN/SFN $-21.2 \%$, SUSP $-83.1 \%$. These results correspond to the data of recent studies [20]. This discrepancy might have resulted from continuous improvement in FNA technique, since the data for the Bethesda system were collected several years ago. Differences may also relate to random variation or institutional differences in pathologic interpretation [11]. Because the incidence of 
malignancy within indeterminate lesions ranges varies widely, there are no clear guidelines for the management of these lesions [21].

Recently, molecular testing of FNA biopsy specimens for genetic alterations frequently associated with TC has emerged as another valuable diagnostic tool [22]. Several prospective studies reported that by molecular testing of thyroid nodules for BRAF, RAS, RET/ PTC, and PAX8/PPARy mutations in cytological material, the difference of any mutation was a strong indicator of cancer because about $97 \%$ of mutation-positive nodules had a malignant diagnosis at histology [16].

Among the various oncogenic events identified in PTC, BRAF V600E is the most common mutation, it ranges from 30 to $80 \%$ (depending on the geographical region) $[6,22]$. Because the BRAF V600E mutation has a high specificity ( $99 \%$ ) and high positive predictive value, it can be used to improve the diagnostic accuracy of US-guided FNA and to overcome the limitations of US-FNA $[3,18]$. On the other hand, reported low sensitivity of BRAF V600E mutation in indeterminate thyroid nodules dramatically limits the applicability of this molecular marker. Moreover, Naif et al. meta-analysis showed that BRAF V600E mutation analysis of FNA cytology has only $30 \%$ sensitivity for PTC in those cases that were reported as indeterminate [23]. In our analysis, we established a relatively high rate $(67.5 \%$ ) of BRAF-positive PTCs in patients with cytologically indeterminate nodules. This can be partially explained by the fact that in our study BRAF V600E mutation status was established from both, FNA biopsy and histology specimens. Moreover, high rate of BRAF V600E positive cases may be related to the distribution of histological types of PTC. According to literature, BRAF V600E mutation is detected for about $60 \%$ of the classical PTC type cases, and only up to $12 \%$ for patients diagnosed with follicular type of PTC [24]. In our study, 71.4\% patients had classical type of PTC, $24.7 \%$ had follicular type, and $3.9 \%$ had oncocytic type. BRAF V600E mutation was found for $78.2 \%, 42.1 \%$, and $33.3 \%$ patients, respectively. Even we have found relatively high BRAF V600E mutation rate, it is still too low for reliable differentiation between PTC and benign nodular thyroid disease when the mutation is absent.

Several studies investigated cytomorphological features in FNA of indeterminate thyroid nodules, and found an association between malignancy and cytologic atypia, which is frequently described in FNA reports. The malignancy risk of cytologic atypia in indeterminate thyroid nodules varies among different studies between $28 \%$ and $71 \%[17,21,25,26]$. Kato with colleagues hypothesized that individual descriptors (cytomorphological features) of atypia could further differentiate malignant tumors from benign lesions, and therefore compared the risk of malignancy in FNA reports describing atypia to FNAs with no atypia. They established the exact risk of specific atypical cytomorphological features, and suggested that the presence of four or more cytomorphological features in the cytology report, or the presence of nuclear inclusions and grooves together increased the risk of malignancy to $80 \%$ [26]. In our study, we found that the presence of high anisokaryosis, streaked chromatin, dark cytoplasm, lymphocyte infiltration, papillary structures, atypia, intranuclear cytoplasmic inclusions, psammoma bodies, densely colloid, and eosinophilic colloid bodies were significantly related to PTC. Lesions with homogenous chromatin, light cytoplasm, prominent amount of colloid, and liquid colloid were shown to be significantly related to benign nodular thyroid disease. However, cytomorphology of FNA biopsy alone cannot sufficiently differentiate indeterminate thyroid nodules.

To the best of our knowledge, this is the first study to evaluate the use of BRAF V600E mutation status combined with cytomorphological features for diagnosing PTC in indeterminate thyroid nodules. Analysis of BRAF V600E mutation status combined with cytomorphological features by the logistic regression model determined that out of all cytomorphological features significant for PTC diagnosis were only: liquid colloid consistency (indirect dependency of PTC diagnosis), papillary structures (direct dependency of PTC diagnosis), and eosinophilic colloid bodies (direct dependency of PTC diagnosis). The estimated sensitivity of our diagnostic model was $-80.5 \%$, specificity - $99.3 \%$, positive predictive value $-98.4 \%$, negative predictive value $-90.4 \%$, and accuracy $92.7 \%$.

However, this study has several limitations, which have to be taken into account when interpreting the results. First of all, this is a cohort and monocentral study. Secondly, the study solves only a part of the diagnostic problems, since only most common TC diagnosis (PTC) is considered. For diagnosis of medullary TC measurement of serum calcitonin level is a sensitive and specific test. This test should be performed for all patients, who are suspected for TC. Currently, there are no reliable criteria for diagnosis of follicular TC, but by active surveillance of low risk group patients, the decision on surgical treatment can be made by assessing the dynamic of clinical signs, for example, tumor growth tendencies, changes in the Bethesda cytological category. The fact that part of the BRAF V600E mutation was established from histology specimens can be considered as a limitation of this study, but it also reflects the benefits of patient surveillance and repeated FNA biopsies.

To sum up, our model has diagnostic specification which can help clinicians to make decision for the best therapeutic approach. For the patients without presence of extrathiroidal extension on US examination, normal calcitonin blood level and cytologically indeterminate thyroid nodules who are assigned to a low risk group by our PTC diagnosis probability calculator (available at www.ptc-calc. we2host.lt) the active surveillance can be offered. For these patients, an US examination with a FNA biopsy and BRAF V600E mutation analysis should be repeated. Surgical treatment should be recommended for all patients assigned to a high risk group.

In conclusion, evaluation of BRAF V600E mutation status combined with cytomorphological features for diagnosis of PTC in cytologically indeterminate thyroid nodules can significantly improve diagnostic accuracy and reduce the number of diagnostic operations. Our PTC diagnosis probability calculator for cytologically indeterminate thyroid nodules is available at www.ptc-calc.we2host.lt.

\section{Conflict of Interest}

No conflict of interest has been declared by the authors. 


\section{References}

[1] Gamme G, Parrington T, Wiebe E et al. The utility of thyroid ultrasonography in the management of thyroid nodules. Can J Surg 2017; 60: 134-139

[2] Jameson L]. Minimizing unnecessary surgery for thyroid nodules. N Engl J Med 2012; 367: 765-767

[3] Haugen BR, Alexander EK, Bible KC et al. 2015 American thyroid association management guidelines for adult patients with thyroid nodules and differentiated thyroid cancer: The american thyroid association guidelines task force on thyroid nodules and differentiated thyroid cancer. Thyroid 2016; 26: 1-133

[4] Davies L, Randolph G. Evidence-based evaluation of the thyroid nodule. Otolaryngol Clin North Am 2014; 47: 461-474

[5] Kitahara CM, McCullough ML, Franceschi S et al. Anthropometric factors and thyroid cancer risk by histological subtype: pooled analysis of 22 prospective studies. Thyroid 2016; 26: 306-318

[6] Omur O, Baran Y. An update on molecular biology of thyroid cancers. Crit Rev Oncol Hematol 2014; 90: 233-252

[7] Deniwar A, Hambleton C, Thethi T et al. Examining the Bethesda criteria risk stratification of thyroid nodules. Pathol Res Pract 2015; 211: $345-348$

[8] Cibas ES, Ali SZ. The Bethesda system for reporting thyroid cytopathology. Am J Clin Pathol 2009; 132: 658-665

[9] Ohori NP, Singhal R, Nikiforova MN et al. BRAF mutation detection in indeterminate thyroid cytology specimens: Underlying cytologic, molecular, and pathologic characteristics of papillary thyroid carcinoma. Cancer Cytopathol 2013; 121: 197-205

[10] Lastra RR, Pramick MR, Crammer C] et al. Implications of a suspicious afirma test result in thyroid fine-needle aspiration cytology: An institutional experience. Cancer Cytopathol 2014; 122: 737-744

[11] Beiša A, Kvietkauskas $M$, Beiša $V$ et al. The utility of the Bethesda category and its association with BRAF mutation in the prediction of papillary thyroid cancer stage. Langenbecks Arch Surg 2017; 402: 227-234

[12] Dağlar-Aday A, Toptaş B, Oztürk T et al. Investigation of BRAF V600E mutation in papillary thyroid carcinoma and tumor-surrounding nontumoral tissues. DNA Cell Biol 2013; 32: 13-18

[13] Hay ID, Thompson GB, Grant CS et al. Papillary thyroid carcinoma managed at the Mayo Clinic during six decades (1940-1999): Temporal trends in initial therapy and long-term outcome in 2444 consecutively treated patients. World J Surg 2002; 26: 879-885
[14] Zoghlami A, Roussel F, Sabourin JC et al. BRAF mutation in papillary thyroid carcinoma: Predictive value for long-term prognosis and radioiodine sensitivity. Eur Ann Otorhinolaryngol Head Neck Dis 2014; 131: 7-13

[15] Brace MD, Wang J, Petten M et al. Differential expression of transforming growth factor-beta in benign vs. papillary thyroid cancer nodules; a potential diagnostic tool? J Otolaryngol Head Neck Surg 2014; 43: 22

[16] Pacini F, Castagna MG, Brilli L et al. Thyroid cancer: ESMO Clinical Practice Guidelines for diagnosis, treatment and follow-up. Ann Oncol 2010; 21: 214-219

[17] Meng Z, Lu J, Wu H et al. Mutant-specific BRAF and CD117 immunocytochemistry potentially facilitate risk stratification for papillary thyroid carcinoma in fine-needle aspiration biopsy specimens. Tumour Biol 2016; 37: 611-618

[18] Seo JY, Kim EK, Kwak JY. Additional BRAF mutation analysis may have additional diagnostic value in thyroid nodules with "suspicious for malignant" cytology alone even when the nodules do not show suspicious US features. Endocrine 2014; 47: 283-289

[19] Rodrigues HG, de Pontes AA, Adan LF. Use of molecular markers in samples obtained from preoperative aspiration of thyroid. Endocr J 2012; 59: 417-424

[20] Ho AS, Sarti EE, Jain KS et al. Malignancy rate in thyroid nodules classified as Bethesda category III (AUS/FLUS). Thyroid 2014; 24 832-839

[21] Miller B, Burkey S, Lindberg G et al. Prevalence of malignancy within cytologically indeterminate thyroid nodules. Am J Surg 2004; 188: 459-462

[22] Yip L, Nikiforova MN, Carty SE et al. Optimizing surgical treatment of papillary thyroid carcinoma associated with BRAF mutation. Surgery 2009; 146: 1215-1223

[23] Fnais N, Soobiah C, Al-Qahtani K et al. BRAFV600E mutation as a predictor of thyroid malignancy in indeterminate nodules: $\mathrm{A}$ systematic review and meta-analysis. Hum Pathol 2015; 46: 1443-1454

[24] Xing M. BRAF mutation in thyroid cancer. Endocr Relat Cancer 2005; 12: 245-262

[25] Kelman AS, Rathan A, Leibowitz ] et al. Thyroid cytology and the risk of malignancy in thyroid nodules: importance of nuclear atypia in indeterminate specimens. Thyroid 2001; 11: 271-277

[26] Kato MA, Buitrago D, Moo TA et al. Predictive value of cytologic atypia in indeterminate thyroid fine-needle aspirate biopsies. Ann Surg Oncol 2011; 18: 2893-2898 